FIRENZE-DDF-392/07/02

\title{
The linear BESS model and double Higgs-strahlung production
}

\author{
R. Casalbuoni and L. Marconi \\ Dipartimento di Fisica, Università di Firenze, I-50125 Firenze, Italia \\ I.N.F.N., Sezione di Firenze, I-50125 Firenze, Italia
}

\begin{abstract}
In this paper we evaluate, in the context of the linear BESS model, the cross-section for the double Higgs-strahlung process. We find that, within the bounds given by the actual experimental data, significant deviations with respect to the SM may arise. In the linear BESS model not only the self-couplings of the Higgs are modified, but also the $Z$-Higgs couplings. We think that this is a generic feature of any extension of the SM and, in our opinion, it should be kept in mind in analyzing the future data on the process studied here.
\end{abstract}

\section{Introduction}

During the last decade the Standard Model (SM) has been verified with great accuracy and today there is no clear evidence for any deviation of the experimental data from the theoretical expectations. However, as it is well known, there is more than one reason to look for extensions of the SM. The main scenarios considered for such extensions are supersymmetry [1] and technicolor [2]. The last type of models are somewhat disfavored, considered the experimental data, mainly because usually there is no natural limit in which they reduce to the SM. On the other hand the supersymmetric models are generally such that in the limit of heavy supersymmetric partners of the SM particles one gets the SM. This property is called decoupling. The linear BESS model, although belonging naturally to a technicolor type scenario, enjoys the decoupling property [3. As such it turns out to be compatible with the actual experimental data. In the model there are six new heavy vector bosons and two heavy scalars and it is characterized by a heavy scale of order of a few $\mathrm{TeV}$. Decoupling the heavy particles, as we will do in 
this paper in order to get an effective lagrangian for the SM particles, has quite different results in the low-energy physics and in the physics associated with the standard Higgs. As far as low-energy is concerned the effects of new physics are very soft leading to corrections $\mathcal{O}\left(1 / u^{2}\right)$ ( $u$ being the heavy scale). However there are potentially significant corrections to the Higgs potential and to the couplings of the Higgs to the $Z$. Therefore in this work we study the process of double Higgs-strahlung production where all these couplings are present [4. This process will be relevant at the future $e^{+} e^{-}$colliders: in $e^{+} e^{-}$collisions the leading electroweak processes for double Higgs production are $e^{+} e^{-} \longrightarrow Z H H$ (double Higgs- strahlung) and $e^{+} e^{-} \longrightarrow \bar{\nu}_{e} \nu_{e} H H$ (fusion mechanism). The second process is relevant for C.M. energy higher than $0.6 \mathrm{TeV}+4 M_{H}$ [5]. In this paper we will be interested at the physical capabilities of TESLA [6] and therefore we will consider only the first process, the relevant energy at TESLA being of the order of $500 \mathrm{GeV}$. In this paper we will take into account corrections to the SM cross-section arising from the couplings of the Higgs to the $Z$ boson since, as we shall see, these are important in the linear BESS model. We think that this is an important point, since it is difficult to imagine a generic model where the only difference with respect to the SM is in the Higgs self-coupling. In fact modifications of all these couplings with respect to the ones of the SM appear naturally as contributions of dimension-6 operators [7].

We have organized the paper starting in Section 2 with a presentation of the cross-section for the double Higgs-strahlung, where we leave all the Higgs couplings arbitrary for the reasons explained above. In Section 3 we give the expressions for the Higgs self-coupling and for the relevant $Z$ Higgs couplings within the linear BESS model. These couplings, as already said, have been evaluated by eliminating all the heavy fields from the BESS lagrangian obtaining an effective low-energy description of the SM fields. Finally, in Section 4, we present our numerical results showing that, under the constraint of compatibility with the low-energy and LEP/SLC data, significant deviations from the SM expectation of the double Higgs-strahlung cross-section might arise.

\section{Double Higgs-strahlung production}

We give here the expression for the unpolarized differential cross-section for the process of double Higgs-strahlung $e^{+} e^{-} \longrightarrow Z H H$ [8] assuming arbitrary 
trilinear Higgs self-coupling, $\lambda_{H H H}$, and couplings $\lambda_{H Z Z}$ and $\lambda_{H H Z Z}$ of the Higgs to the $Z$ boson. After integration over the angular variables one gets:

$$
\left(\frac{d \sigma}{d x_{1} d x_{2}}\right)=\left(\frac{d \sigma_{0}}{d x_{1} d x_{2}}+\frac{d \sigma_{1}}{d x_{1} d x_{2}}+\frac{d \sigma_{2}}{d x_{1} d x_{2}}+\frac{d \sigma_{3}}{d x_{1} d x_{2}}\right)+\left(y_{1} \leftrightarrow y_{2}\right),
$$

where:

$$
\begin{aligned}
\frac{d \sigma_{0}}{d x_{1} d x_{2}} & =\frac{\sqrt{2} G_{F}^{3} M_{Z}^{6}}{384 \pi^{3}} \frac{\left(2 c_{V}\right)^{2}+\left(2 c_{A}\right)^{2}}{s\left(1-\mu_{Z}\right)^{2}} \frac{2}{G_{F}^{2} M_{Z}^{8}} a^{2} f_{0}, \\
\frac{d \sigma_{1}}{d x_{1} d x_{2}} & =\frac{\sqrt{2} G_{F}^{3} M_{Z}^{6}}{384 \pi^{3}} \frac{\left(2 c_{V}\right)^{2}+\left(2 c_{A}\right)^{2}}{s\left(1-\mu_{Z}\right)^{2}} \frac{\lambda_{H Z Z}^{4}}{2 G_{F}^{2} M_{Z}^{8}} \frac{f_{1}}{4 \mu_{Z}\left(y_{1}+\mu_{H Z}\right)^{2}}, \\
\frac{d \sigma_{2}}{d x_{1} d x_{2}} & =\frac{\sqrt{2} G_{F}^{3} M_{Z}^{6}}{384 \pi^{3}} \frac{\left(2 c_{V}\right)^{2}+\left(2 c_{A}\right)^{2}}{s\left(1-\mu_{Z}\right)^{2}} \frac{\lambda_{H Z Z}^{4}}{2 G_{F}^{2} M_{Z}^{8}} \frac{f_{2}}{4 \mu_{Z}\left(y_{1}+\mu_{H Z}\right)\left(y_{2}+\mu_{H Z}\right)} \\
\frac{d \sigma_{3}}{d x_{1} d x_{2}} & =\frac{\sqrt{2} G_{F}^{3} M_{Z}^{6}}{384 \pi^{3}} \frac{\left(2 c_{V}\right)^{2}+\left(2 c_{A}\right)^{2}}{s\left(1-\mu_{Z}\right)^{2}} \frac{\lambda_{H Z Z}^{2}}{G_{F}^{2} M_{Z}^{8}} \frac{a f_{3}}{2\left(y_{1}+\mu_{H Z}\right)} .
\end{aligned}
$$

We have introduced the scaled energies of the two Higgs particles $x_{1,2}=$ $2 E_{1,2} / \sqrt{s}, x_{3}=2-x_{1}-x_{2}$ the scaled energy of the $Z$ boson and $y_{i}=1-x_{i}$. We have also introduced scaled square masses $\mu_{i}=M_{i}^{2} / s, i=Z, H$, and $\mu_{i j}=\mu_{i}-\mu_{j}$. We have left unspecified the vector and axial coupling of the $Z$ to the fermions, $c_{V}$ and $c_{A}$. The coefficient $a$ turns out to be:

$$
a=\frac{3 \lambda_{H H H} \lambda_{H Z Z}}{y_{3}-\mu_{H Z}}+\frac{\lambda_{H Z Z}^{2}}{y_{1}+\mu_{H Z}}+\frac{\lambda_{H Z Z}^{2}}{y_{2}+\mu_{H Z}}+\lambda_{H H Z Z} s
$$

and the coefficients $f_{i}$ are:

$$
\begin{aligned}
f_{0}= & \mu_{Z}\left[\left(y_{1}+y_{2}\right)^{2}+8 \mu_{z}\right] / 8, \\
f_{1}= & \left(y_{1}-1\right)^{2}\left(\mu_{Z}-y_{1}\right)^{2}-4 \mu_{H} y_{1}\left(y_{1}+y_{1} \mu_{Z}-4 \mu_{Z}\right)+ \\
& +\mu_{Z}\left(\mu_{Z}-4 \mu_{H}\right)\left(1-4 \mu_{H}\right)-\mu_{Z}^{2}, \\
f_{2}= & {\left[\mu_{Z}\left(y_{3}+\mu_{Z}-8 \mu_{H}\right)-\left(1+\mu_{Z}\right) y_{1} y_{2}\right]\left(1+y_{3}+2 \mu_{Z}\right)+} \\
& +y_{1} y_{2}\left[y_{1} y_{2}+1+\mu_{Z}^{2}+4 \mu_{H}\left(1+\mu_{Z}\right)\right]+4 \mu_{H} \mu_{Z}\left(1+\mu_{Z}+4 \mu_{H}\right)+\mu_{Z}^{2}, \\
f_{3}= & y_{1}\left(y_{1}-1\right)\left(\mu_{Z}-y_{1}\right)-y_{2}\left(y_{1}+1\right)\left(y_{1}+\mu_{Z}\right)+ \\
& +2 \mu_{Z}\left(1+\mu_{Z}-4 \mu_{H}\right) .
\end{aligned}
$$

Each term in Eq. (21) is easily identified by looking at the propagators and couplings. For instance, the square of the first term in the parameter $a$ 
of Eq. (3) corresponds to the square of the diagram which involves the trilinear Higgs coupling $\lambda_{H H H}$. The other terms are related to sequential Higgs-strahlung amplitudes and the four-gauge-Higgs boson coupling.

As we have said we have left unspecified the various couplings appearing in the differential cross-section. The reason is that for any specified model, all the couplings are generally different with respect to the ones of the SM, although some of the couplings, as for instance, $c_{V}$ and $c_{A}$ are strongly constrained by the LEP and SLC data. In the next Section we will consider a model for which the parameters can be chosen in such a way to respect the experimental bounds, but nevertheless able to produce strong deviations in the cross-section we are considering here.

\section{The linear BESS model}

The linear BESS model [3] is an extension of the SM involving six more vector bosons, $V_{L}^{i}$ and $V_{R}^{i}, i=1,2,3$, and two more scalar particles $\rho_{L}$ and $\rho_{R}$. The light scalar field, the usual Higgs, will be denoted here by $\rho_{U}$. Furthermore it is characterized by a large mass scale, $u$, such that for $u \rightarrow \infty$ all the new particles decouple from the SM modes. Therefore, by appropriate choice of the scale, it is possible to satisfy the experimental bounds from low energy and LEP/SLC experiments. The masses of the new particles are of order $u$ (heavy particles), and the deviations from the SM can be discussed in terms of the parameter expansion $v^{2} / u^{2}\left(v^{2}=1 / \sqrt{2} G_{F}\right)$. The new particles modify the double Higgs-strahlung cross-section since they are coupled to the particles of the SM (see [3]). Therefore there are many more diagrams involved in this process than the ones coming from the SM alone. A way of treating this problem is to use an effective lagrangian describing the linear BESS model at energies lower than the heavy scale $u$. This lagrangian can be evaluated solving the classical equations of motion for the heavy fields in the low-energy limit. The full lagrangian from which the classical equations are extracted is given in [3]. The effective lagrangian obtained in this way has exactly the same structure of the SM lagrangian, except that the couplings are modified by the heavy physics. We give here the results of this calculation concerning the couplings which are relevant in the process we are interested in. These are nothing but the couplings appearing in eqs. (22) and (3). First we recall the expressions for the couplings in the SM: 


$$
\begin{gathered}
\lambda_{H H H_{S M}}=\frac{1}{2} \sqrt{\sqrt{2} G_{F}} M_{H}^{2}, \\
\lambda_{H Z Z_{S M}}=\frac{1}{4 \sqrt{\sqrt{2} G_{F}}} \frac{e^{2}}{s_{\theta_{W}}^{2} c_{\theta_{W}}^{2}}, \quad \lambda_{H H Z Z_{S M}}=\frac{1}{8} \frac{e^{2}}{s_{\theta_{W}}^{2} c_{\theta_{W}}^{2}}, \\
c_{V_{S M}}=-\frac{1}{2}+2 s_{\theta_{W}}^{2}, \quad c_{A_{S M}}=-\frac{1}{2},
\end{gathered}
$$

where $s_{\theta_{W}}$ is defined by:

$$
s_{\theta_{W}}^{2}=\frac{1}{2}-\sqrt{\frac{1}{4}-\frac{\pi \alpha}{\sqrt{2} G_{F} M_{Z}^{2}}} .
$$

Then we find:

- $H H H: \lambda_{H H H_{S M}}\left(1-\Delta_{H H H} \epsilon\right)$,

- $H Z Z: \lambda_{H Z Z_{S M}}\left(1-\Delta_{H Z Z} \epsilon\right)$,

- HHZZ: $\lambda_{H H Z Z_{S M}}\left(1-\Delta_{H H Z Z} \epsilon\right)$,

- $\Psi \bar{\Psi} Z:-\frac{e}{s_{\theta_{W}} c_{\theta_{W}}} \gamma^{\mu} \frac{1}{2}\left[c_{V_{S M}}+\Delta c_{V} \epsilon-\left(c_{A_{S M}}+\Delta c_{A} \epsilon\right) \gamma_{5}\right]$

where $\epsilon=v^{2} / u^{2}$ is our expansion parameter and:

$$
\begin{aligned}
\Delta_{H H H} & =3 q^{2} \\
\Delta_{H Z Z} & =q^{2}+\frac{e^{4}}{g_{2}^{4}} \frac{1-2 c_{\theta_{W}}^{2}+2 c_{\theta_{W}}^{4}}{s_{\theta_{W}}^{4} c_{\theta_{W}}^{4}} \\
\Delta_{H H Z Z} & =4 q^{2}+\frac{e^{4}}{9 c_{\theta_{W}}^{4}+s_{\theta_{W}}^{4}}+\frac{e^{5}}{s_{\theta_{W}}^{4} c_{\theta_{W}}^{4}} \frac{8}{s_{\theta_{W}} c_{\theta_{W}}^{4}}, \\
\Delta c_{V} & =\frac{1}{4} \frac{e^{4}}{g_{2}^{4}} \frac{3-16 c_{\theta_{W}}^{2}+18 c_{\theta_{W}}^{4}-4 c_{\theta_{W}}^{6}}{s_{\theta_{W}}^{4} c_{\theta_{W}}^{4}\left(c_{\theta_{W}}^{2}-s_{\theta_{W}}^{2}\right)}, \\
\Delta c_{A} & =\frac{1}{4} \frac{e^{4}}{g_{2}^{4}} \frac{s_{\theta_{W}}^{4}+c_{\theta_{W}}^{4}}{s_{\theta_{W}}^{4} c_{\theta_{W}}^{4}},
\end{aligned}
$$

where $g_{2}$ is the gauge coupling of the fields $V_{L}$ and $V_{R}$, whereas $q$ is a parameter appearing in the scalar sector of the BESS model [3]:

$$
\begin{aligned}
V^{\text {Higgs }}= & 2 \mu^{2}\left(\rho_{L}^{2}+\rho_{R}^{2}\right)+\lambda\left(\rho_{L}^{4}+\rho_{R}^{4}\right)+2 m^{2} \rho_{U}^{2}+h \rho_{U}^{4}+ \\
& +2 f_{3} \rho_{L}^{2} \rho_{R}^{2}+2 f \rho_{U}^{2}\left(\rho_{L}^{2}+\rho_{R}^{2}\right),
\end{aligned}
$$


with $q$ defined as:

$$
q=\frac{f}{f_{3}+\lambda},
$$

We see that $q$ is the ratio among the coupling of the heavy scalar fields to the light one and the sum of the quartic couplings of heavy scalar fields. Therefore it is the essential parameter (together with the ratio of the two expectation values $v / u$ ) which determines the mixing of the physical Higgs field to the heavy Higgs fields $\rho_{L}$ and $\rho_{R}$. Although $q$ is a free parameter of the model, we expect it to be of order one in absence of any fine tuning of the couplings in the Higgs sector.

It is important to notice that $M_{H}$ and $q$ are the only new parameters appearing in this analysis with respect to the analysis of the LEP data. Also notice that $q$ modifies the $H H H$ coupling and the couplings $H Z Z$ and $H H Z Z$. This is quite important because it shows that fitting the data to a theoretical form of the cross-section, where only the trilinear coupling of the Higgs is left as a free parameter, might not be the right thing to be done. In fact this example shows the possibility of correlations among different Higgs couplings.

It will be convenient to trade the parameters $M_{H}, \epsilon, g_{2}$ and $q$ with the set:

$$
M_{H}, \frac{M_{W}}{M_{V}}, \frac{g_{S M}}{g_{2}}, q .
$$

In particular:

$$
\frac{M_{W}^{2}}{M_{V}^{2}}=\epsilon \frac{g_{S M}^{2}}{g_{2}^{2}},
$$

where $M_{V}$ is the mass scale of the new vector bosons. We will consider the expansion of the cross-section around its SM value. For this we need to keep under control the parameter $q$. From eqs. (7) and (8) we find that the condition $q$ must satisfy is:

$$
4 q^{2} \epsilon \lesssim 1 \quad \Longrightarrow \quad q \lesssim \frac{1}{2} \frac{g_{S M}}{g_{2}} \frac{M_{V}}{M_{W^{ \pm}}} .
$$




\section{Numerical results}

At first order in the expansion parameter $\epsilon=v^{2} / u^{2}$ the difference between the SM and BESS differential cross-section is:

$$
\begin{aligned}
& \Delta\left(\frac{d \sigma}{d x_{1} d x_{2}}\right)=\frac{\sqrt{2} G_{F}^{3} M_{Z}^{6}}{384 \pi^{3} s\left(1-\mu_{Z}\right)^{2}} \frac{1}{G_{F}^{2} M_{Z}^{8}} \cdot \\
& \cdot\left\{2 f_{0}\left[8\left(c_{V_{S M}} \Delta c_{V}+c_{A_{S M}} \Delta c_{A}\right) a^{2}-2 a a_{1}\left(\left(2 c_{V_{S M}}\right)^{2}+\left(2 c_{A_{S M}}\right)^{2}\right)\right]+\right. \\
& +\frac{1}{4 \mu_{Z}\left(y_{1}+\mu_{H Z}\right)}\left(\frac{f_{1}}{y_{1}+\mu_{H Z}}+\frac{f_{2}}{y_{2}+\mu_{H Z}}\right) \frac{\lambda_{H Z Z_{S M}}^{4}}{2} \cdot \\
& \cdot\left[8\left(c_{V_{S M}} \Delta c_{V}+c_{A_{S M}} \Delta c_{A}\right)-4 \Delta \lambda_{H Z Z}\left(\left(2 c_{V_{S M}}\right)^{2}+\left(2 c_{A_{S M}}\right)^{2}\right)\right]+ \\
& +\frac{f_{3}}{2\left(y_{1}+\mu_{H Z}\right)} \lambda_{H Z Z_{S M}}^{2}\left[8\left(c_{V_{S M}} \Delta c_{V}+c_{A_{S M}} \Delta c_{A}\right) a-\left(a_{1}+2 a \Delta \lambda_{H Z Z}\right)\left(\left(2 c_{V_{S M}}\right)^{2}+\right.\right. \\
& \left.\left.\left.+\left(2 c_{A_{S M}}\right)^{2}\right)\right]\right\} \frac{M_{W^{ \pm}}^{2}}{M_{V}^{2}} \frac{1}{\left(g_{S M} / g_{2}\right)^{2}},
\end{aligned}
$$

where $a, f_{i}$ are defined in Eq. (3), (41), and $a_{1}$ is given by

$$
\begin{aligned}
a_{1}= & \frac{3 \lambda_{H H H_{S M}} \lambda_{H Z Z_{S M}}\left(\Delta \lambda_{H H H}+\Delta \lambda_{H Z Z}\right)}{y_{3}-\mu_{H Z}}+\frac{2 \lambda_{H Z Z_{S M}}^{2} \Delta \lambda_{H Z Z}}{y_{1}+\mu_{H Z}}+ \\
& +\frac{2 \lambda_{H Z Z_{S M}}^{2} \Delta \lambda_{H Z Z}}{y_{2}+\mu_{H Z}}+\lambda_{H H Z Z_{S M}} \Delta \lambda_{H H Z Z} s .
\end{aligned}
$$

The analysis made by TESLA [6] (see Table 1) shows that the estimated un-

\begin{tabular}{|l|c|c|c|}
\hline$M_{H}(\mathrm{GeV})$ & 120 & 130 & 140 \\
\hline \hline$N_{H H Z}$ & 80 & 64 & 44 \\
Efficiency & 0.43 & 0.43 & 0.49 \\
\hline$\delta \sigma / \sigma$ & \pm 0.17 & \pm 0.19 & \pm 0.23 \\
\hline
\end{tabular}

Table 1: Number of events, efficiency and cross-section uncertainty of double Higgs-strahlung production with an integrated luminosity of $1000 \mathrm{fb}^{-1}$ [6] .

certainty in measuring the total cross-section for the double Higgs-strahlung is around $20 \%$. Therefore, the region in the parameter space where we may expect to find deviations to the SM within the model considerd here is the 
region complementary to the one defined by the inequality

$$
\left|\frac{\Delta \sigma}{\sigma_{S M}}\right|=\left|\frac{\sigma_{B E S S}-\sigma_{S M}}{\sigma_{S M}}\right| \leq 0.2 .
$$

The linear BESS parameter space gets limitations from the LEP and the other low energy data only in the sector $\left(M_{V}, g_{S M} / g_{2}\right)$ 9. The results of this analysis, which has been made using the data of ref. [10, are given in Fig. 1 , where the $95 \%$ CL allowed region is shown. Using these results we may

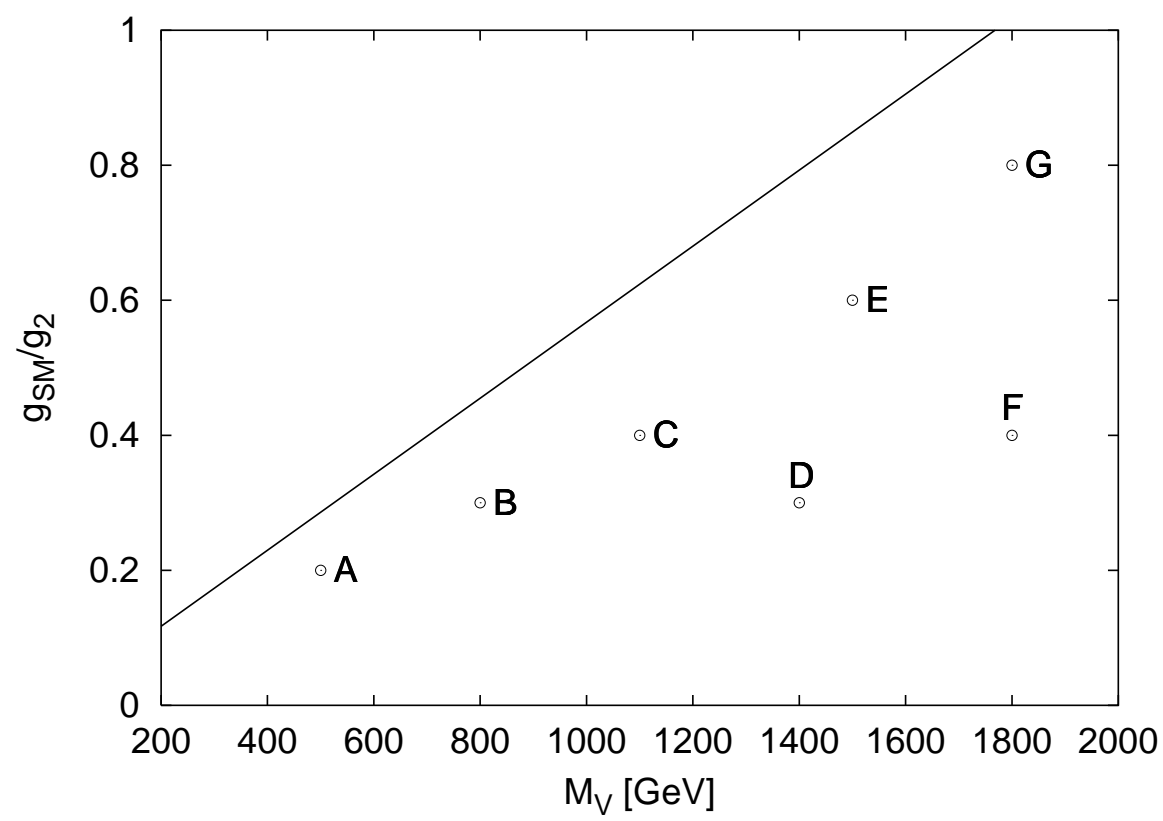

Figure 1: $95 \% C L$ allowed region in the plane $\left(M_{V}, g_{S M} / g_{2}\right)$ from low-energy and $L E P / S L C$ data. We have shown the points chosen for the present analysis.

eliminate the parameters $\left(M_{V}, g_{S M} / g_{2}\right)$ from our analysis by choosing some representative point within the allowed region. Therefore we have selected seven points ranging in the allowed region in a range of heavy vector masses between 500 and $1800 \mathrm{GeV}$. The only remaining free parameters are now $q$ and $M_{H}$. However one can see that for $110 \leq M_{H} \lesssim 200 \mathrm{GeV}$, that is up to the kinematical limit for producing two Higgs particles at TESLA energy of $500 \mathrm{GeV}$, the deviations do not depend on $M_{H}$. It follows that in this range of values of $M_{H}$ the only relevant parameter of the model is $q$. In Fig. 2 
we have plotted the variation of the ratio $\Delta \sigma_{S M}$ with $q$ for the points shown in Fig. 1. From this plot, for each point of Fig. 1, we may determine the minimal value of $q$ (here denoted by $q_{M I N}$ ) such that for $q>q_{M I N}$ TESLA will be able to appreciate deviations from the SM. The numerical values of $q_{M I N}$ obtained in Fig. 2 are reported also in Table 2 where we have also given the limiting values $q_{\text {lim }}$ from eq. (13) showing that our approximation in the expansion of the differential cross-section is certainly valid up to and somewhat above $q_{M I N}$. Also, since we expect $q$ to be naturally of order 1 ,

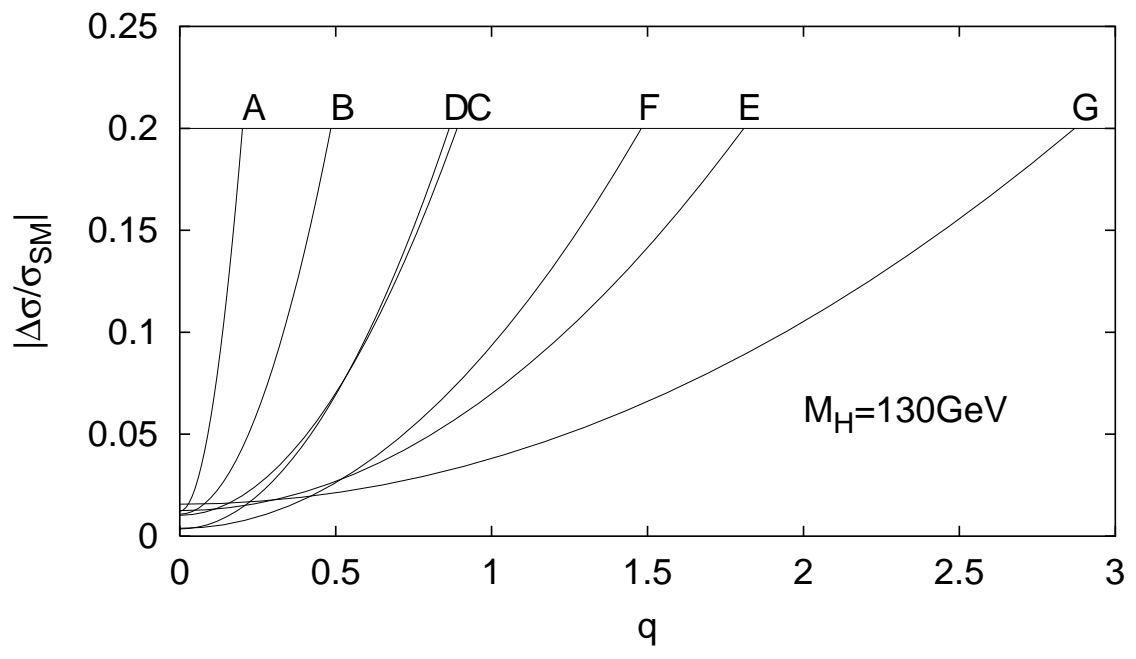

Figure 2: For $\sqrt{s}=500 \mathrm{GeV}$ and $M_{H}=130 \mathrm{GeV}$, we plot $\left|\Delta \sigma / \sigma_{S M}\right|$ as a function of $q$ for each point chosen in the plane $\left(M_{V}, g_{S M} / g_{2}\right)$.

this Table shows that we may expect that TESLA will be able to show the deviations given by the linear BESS model for a range of values of $g_{S M} / g_{2}$ and $M_{V}$ not too high. Typically for $g_{S M} / g_{2} \lesssim 0.5$ and $M_{V} \lesssim 1.5 \mathrm{TeV}$.

In our numerical analysis we have also varied the TESLA energy from $500 \mathrm{GeV}$ to $1 \mathrm{TeV}$ but the results are practically insensitive to this variation of the energy.

A natural question is how radiative corrections affect the results obtained here. The radiative corrections related to the SM particles are the same in the linear BESS model and in the SM. Therefore they cancel in the deviation. These corrections will be important only when the prediction of the BESS model will be compared with the experimental data, but of course, they are 


\begin{tabular}{|c||c|r|c||c|}
\hline & $g_{S M} / g_{2}$ & $M_{V}(\mathrm{GeV})$ & $q_{\text {lim }}$ & $q_{M I N}$ \\
\hline \hline $\mathrm{A}$ & 0.2 & 500 & 0.62 & 0.20 \\
\hline $\mathrm{B}$ & 0.3 & 800 & 1.50 & 0.48 \\
\hline $\mathrm{C}$ & 0.4 & 1100 & 2.73 & 0.88 \\
\hline $\mathrm{D}$ & 0.3 & 1400 & 2.61 & 0.85 \\
\hline $\mathrm{E}$ & 0.6 & 1500 & 5.60 & 1.80 \\
\hline $\mathrm{F}$ & 0.4 & 1800 & 4.48 & 1.47 \\
\hline $\mathrm{G}$ & 0.8 & 1800 & 8.95 & 2.86 \\
\hline
\end{tabular}

Table 2: For each point chosen in the plane $\left(M_{V}, g_{S M} / g_{2}\right)$ we compare the value $q_{\text {lim }}$ (see text) with the maximal value of $q\left(q_{M I N}\right)$ compatible with the $S M$.

perfectly known. The other radiative corrections related to the exchange of heavy particles are a small fraction of the tree-level corrections considered in this paper, and they can safely be ignored in the present analysis.

\section{Conclusions}

In this paper we have evaluated the cross-section for the double Higgsstrahlung in the context of the linear BESS model. The main property of this model is decoupling. This makes possible the compatibility of the model with the actual experimental data. Decoupling derives from the presence in the model of a heavy scale $u$ (of the order or larger than some $T e V$ ) such that the deviations (as far as the low-energy and LEP/SLC physics are concerned) with respect to the SM are of the order $v^{2} / u^{2}$. The model makes clear that new physics may affect the Higgs sector in without touching the low energy phenomenology and, more important, has its effects in all the Higgs couplings. That is, not only the self-coupling, but also the couplings of the Higgs to the $Z$. This, of course, creates correlations in the amplitudes of processes like the one studied here, and this fact should be taken in due care in all future analysis.

As a final comment we notice that since the model presented here decouples at LEP energies, the only way to test it is either to go to the $\mathrm{TeV}$ scale where the new heavy particles should be produced, or to look at Higgs physics, as done in this paper, since the new coupling $q$, involved here, is not relevant at LEP energies. However, as far as the second possibility is 
concerned, it should be stressed that presently we don't know any way of disentangle this model from possible other models with similar decoupling features.

\section{References}

[1] H.P. Nilles, Phys.Rep. 110 (1984) 1; H. Haber and G. Kane, Phys.Rev. D20 (1979) 2619; E. Fahri and L. Susskind, Phys.Rep. 74 (1981) 277; R. Barbieri, Riv. Nuovo Cimento 11 (1988) 1; R. Barbieri, F. Caravaglios and M. Frigeni, Phys.Lett. B279 169.

[2] S. Weinberg, Phys.Rev. D13 (1976) 974; L. Susskind, Phys.Rev. D20 (1979) 2691; S. Dimopoulos and L. Susskind, Nucl.Phys. B155 (1979) 237; E. Eitchen and K. Lane, Phys.Lett. B90 (1980) 125.

[3] R. Casalbuoni, S. De Curtis, D. Dominici and M. Grazzini, Phys.Rev. D56 (1997) 5731.

[4] The relevance of this process was stressed by G.J. Gounaris, D.Schildknecht and F.M. Renard, Phys. Lett. 83B (1979) 191; erratum 89B (1980) 437.

[5] V. Barger, T. Han, Mod.Phys.Lett. A Vol.5 No.9 (1990) 667.

[6] TESLA Technical Design Report, Part III "Physics at an $e^{+} e^{-}$Linear Collider", TESLA Report 2001 - 23.

[7] W. Kilian, M. Krämer, P.M. Zerwas, Phys.Lett. B381 (1996) 243.

[8] A. Djouadi, W. Kilian, M. Muhlleitner and P.M. Zerwas, Eur.Phys.J. C10 (1999) 27; A. Djouadi, H.E. Haber and P.M. Zerwas, Phys.Lett. B375 (1996) 203.

[9] R. Casalbuoni, D. Dominici, P. Chiappetta, A. Deandrea, S. De Curtis and R. Gatto, Phys.Rev. D56 (1997) 2812. For a definition of the $\epsilon$ parameters, see: G. Altarelli, R. Barbieri and S. Jadach, Nucl.Phys. B369 (1992) 3; G. Altarelli, R. Barbieri and F. Caravaglios, Nucl.Phys. B405 (1993) 3; G. Altarelli and R. Barbieri, Phys.Lett. B253 (1991) 161. 
[10] G. Altarelli, "The Standard Electroweak Theory and Beyond", lectures given at Zuoz Summer School on Phenomenology of Gauge Interactions, CERN-TH/2000-291, hep-ph/0011078 (2000). 